ARTICLE

\title{
Imaging-based spectrometer-less optofluidic biosensors based on dielectric metasurfaces for detecting extracellular vesicles
}

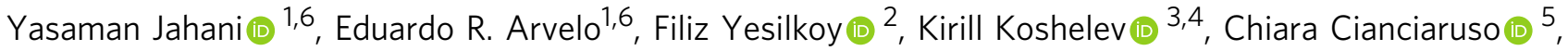 \\ Michele De Palma (i) ${ }^{5}$, Yuri Kivshar (10 ${ }^{3}$ \& Hatice Altug (iD ${ }^{1 \times}$
}

Biosensors are indispensable tools for public, global, and personalized healthcare as they provide tests that can be used from early disease detection and treatment monitoring to preventing pandemics. We introduce single-wavelength imaging biosensors capable of reconstructing spectral shift information induced by biomarkers dynamically using an advanced data processing technique based on an optimal linear estimator. Our method achieves superior sensitivity without wavelength scanning or spectroscopy instruments. We engineered diatomic dielectric metasurfaces supporting bound states in the continuum that allows high-quality resonances with accessible near-fields by in-plane symmetry breaking. The large-area metasurface chips are configured as microarrays and integrated with microfluidics on an imaging platform for real-time detection of breast cancer extracellular vesicles encompassing exosomes. The optofluidic system has high sensing performance with nearly 70 1/RIU figure-of-merit enabling detection of on average 0.41 nanoparticle/ $\mu \mathrm{m}^{2}$ and realtime measurements of extracellular vesicles binding from down to 204 femtomolar solutions. Our biosensors provide the robustness of spectrometric approaches while substituting complex instrumentation with a single-wavelength light source and a complementary-metaloxide-semiconductor camera, paving the way toward miniaturized devices for point-of-care diagnostics.

\footnotetext{
${ }^{1}$ Institute of Bioengineering, École Polytechnique Fédérale de Lausanne (EPFL), Lausanne, Switzerland. ${ }^{2}$ Department of Biomedical Engineering, University of Wisconsin-Madison, Madison, WI, USA. ${ }^{3}$ Nonlinear Physics Center, Research School of Physics, Australian National University, Canberra, Australia. ${ }^{4}$ School of Physics and Engineering, ITMO University, St Petersburg, Russia. ${ }^{5}$ Swiss Institute for Experimental Cancer Research (ISREC), School of Life Sciences, École Polytechnique Fédérale de Lausanne (EPFL), Lausanne, Switzerland. ${ }^{6}$ These authors contributed equally: Yasaman Jahani, Eduardo R. Arvelo. ${ }^{\bowtie}$ email: hatice. altug@epfl.ch
} 
$\mathrm{R}$ eliable, rapid, and highly sensitive detection of disease indicators is crucial for timely and specific diagnostics and treatments $^{1-3}$. Point-of-care (POC) devices are highly desirable for health monitoring and biosafety applications in the field settings as they offer easy-to-use, low-cost and fast detection of numerous indicative biomarkers, such as proteins, antibodies, nucleic acids, and extracellular vesicles, including exosomes, as well as pathogens such as viruses ${ }^{4-7}$. For instance, the 2020 coronavirus outbreak underlines the importance of POC tools that can enable rapid, cost-effective, accurate, and quantitative tests for COVID-19. Optical biosensors based on nanophotonics are eminently sought after as they allow miniaturized lab-on-achip technologies for label-free analysis and multiplexed sensing $4,5,8,9$. When integrated with microfluidics, nanophotonic biosensors can perform automated measurements on low sample volumes and provide quantitative and real-time results without requiring time-consuming and expensive external labels ${ }^{10-13}$. Nanophotonic resonators can enable label-free detection of biomarkers by means of refractometric sensing that exploit optical resonances to monitor the changes in the dielectric properties in the vicinity of the resonators caused by, for instance, the binding of analytes to the surface-immobilized capture molecules. This change in a local refractive index translates into a resonance wavelength shift, which can be employed to quantify analyte binding events. Spectral interrogation is the conventional method to precisely track the resonance wavelength and measure the spectral shift induced by the analyte presence employing commercial spectrometers. The spectroscopic detection has been implemented with numerous nanophotonic resonators, including plasmonic nanoaperture arrays, nanoparticles, and nanoantennas such as rods, tubes, disks, and bow ties ${ }^{13-17}$.

In recent years, there has been a surge of efforts to improve both performance and functionalities of nanophotonic biosensors employing new structures, materials, and devices ${ }^{6,18,19}$. While optimizing the nanostructures for refractometric sensing, one should take into account multiple parameters, including the quality factor $(Q)$ of the resonators, the enhancement factor for the near-field intensities, and the spatial overlap of the enhanced fields with the target analytes. As these parameters tend to be coupled with each other, it is also essential to consider their involved trade-offs (see Supplementary Information note 1). For example, high $Q$ resonances with sharp spectral features are desirable to resolve the spectral shift induced by analyte binding at a superior resolution. However, if the high $Q$ values are achieved by confining the mode tightly inside the nanoresonators, then this can reduce the overlap of the near-fields with the analytes and lower the overall sensitivity. Plasmonic sensors have been taking advantage of large near-field enhancement factors, while dissipative losses in metals lead to low $Q$ resonances, which limits their overall sensing performance ${ }^{20,21}$. Recently, high-index all-dielectric metasurfaces driven by the physics of optical Mie resonances have emerged as an alternative platform as they do not suffer from losses due to the absence of free charges ${ }^{22-31}$. Silicon-based metasurfaces operating within the visible and nearIR (600-900 nm) spectral ranges have been employed for bulk and thin-film sensing using spectrometers ${ }^{32,33}$. Concurrently, studies show that sharp spectral resonances can be achieved by using the concept of bound states in the continuum (BICs), in which the light wave at the resonance remains completely localized in the metasurface even though the state coexists with a continuous electromagnetic spectrum of the environment ${ }^{34,35}$. In practice, BICs are realized with high but finite values of the $Q$ due to structural losses and imperfections and are usually termed "quasi BICs" or "supercavity modes"36,37. The BIC-inspired resonances in the symmetry-broken all-dielectric metasurfaces have recently been used for highly sensitive biosensors ${ }^{38}$.
This earlier work interrogates dielectric metasurfaces supporting quasi-BIC modes using hyperspectral imaging towards highperformance biomolecule detection.

Although spectrometers and wavelength-scanning systems provide reliable spectral shift information for monitoring the changes in the optical response, they are bulky and expensive. In addition, spectrometers are limited in simultaneously collecting data from an array of sensors for implementing multiplexed bioassays. Wavelength-scanning systems can provide spatially resolved spectral information with hyperspectral imaging in a given field of view for monitoring signals from multiple sensing elements ${ }^{38,39}$. However, wavelength-scanning is cumbersome and time-consuming; hence, it cannot be used to gather time-resolved images for measuring molecular binding kinetics. Therefore, there is a need for simple and scalable optical read-out mechanisms that can extract both temporally- and spatially-resolved information from large sensor areas. To this end, red-shifting of the resonance spectrum by the binding of the analytes on the sensor surface can be quantified by tracking the changes in the intensity over a narrow spectral window instead of collecting spectrally resolved information over a broad bandwidth ${ }^{8,40}$. In particular, when combined with a small-scale light source - i.e., laser or light-emitting diode (LED) - and an imaging camera (i.e., complementary metal-oxide-semiconductor (CMOS) or chargecoupled device (CCD) cameras), the intensity-based optical readers can be ultra-compact, low-weight, and portable by eliminating the need for sophisticated spectroscopy instrumentations, including scanning and/or moving parts ${ }^{41}$. Thus, spectrometerless readers can reduce cost and improve mechanical robustness ${ }^{42}$. Furthermore, by acquiring images over a large fieldof-view, they can enable the implementation of microarrays for high-throughput screening applications ${ }^{4}$. For instance, microarrays can be used to detect multiple target analytes from a given sample in a single measurement, saving both time and precious biosamples ${ }^{43}$. Nonetheless, compared to spectral interrogation, intensity interrogation could not achieve sensitive and reliable detection as it is less tolerant of noise factors, such as inhomogeneties in sensors'optical responses and inadequate choice of the light source.

In this work, we introduce single-wavelength imaging-based nanophotonic biosensors for achieving superior sensitivity based on reconstructed spectral shift without cumbersome wavelength scanning and spectrometers. To achieve this goal, we employ quasi-BIC modes manifesting themselves through high- $Q$ resonances of "diatomic metasurfaces" by generalizing and expanding the concept of BIC developed for asymmetric metasurfaces 37 . Diatomic metasurfaces are based on a dimer-type unit cell a.k.a "meta-molecule", in which the dimer symmetry is broken by changing the ellipticity of one of the meta-atoms to support high $Q$ resonance and strong field-analyte overlaps. Compared to single-unit metasurfaces, such diatomic structures provide advanced flexibility in the engineering of the in-plane asymmetry while keeping the design easier to fabricate and make it more feasible for biosensing applications. We combine these metasurfaces with advanced data processing techniques for aided imaging biosensing. Our biosensor acquires large-area intensity images of the metasurface in real-time by using a single wavelength illumination and processes them optimally via a linear estimation algorithm to reconstruct spectral shift data at high accuracy dynamically. For demonstration, we integrated antibody functionalized metasurface chips in a $2 \mathrm{D}$ microarray format with microfluidics and performed in-flow detection and real-time binding of breast cancer extracellular vesicles, which are important biomarkers for diagnostics owing to their connection with disease pathology 44,45 . Our optofluidic sensor has enabled detection of on average 0.41 nanoparticle $/ \mu \mathrm{m}^{2}$ and real-time 

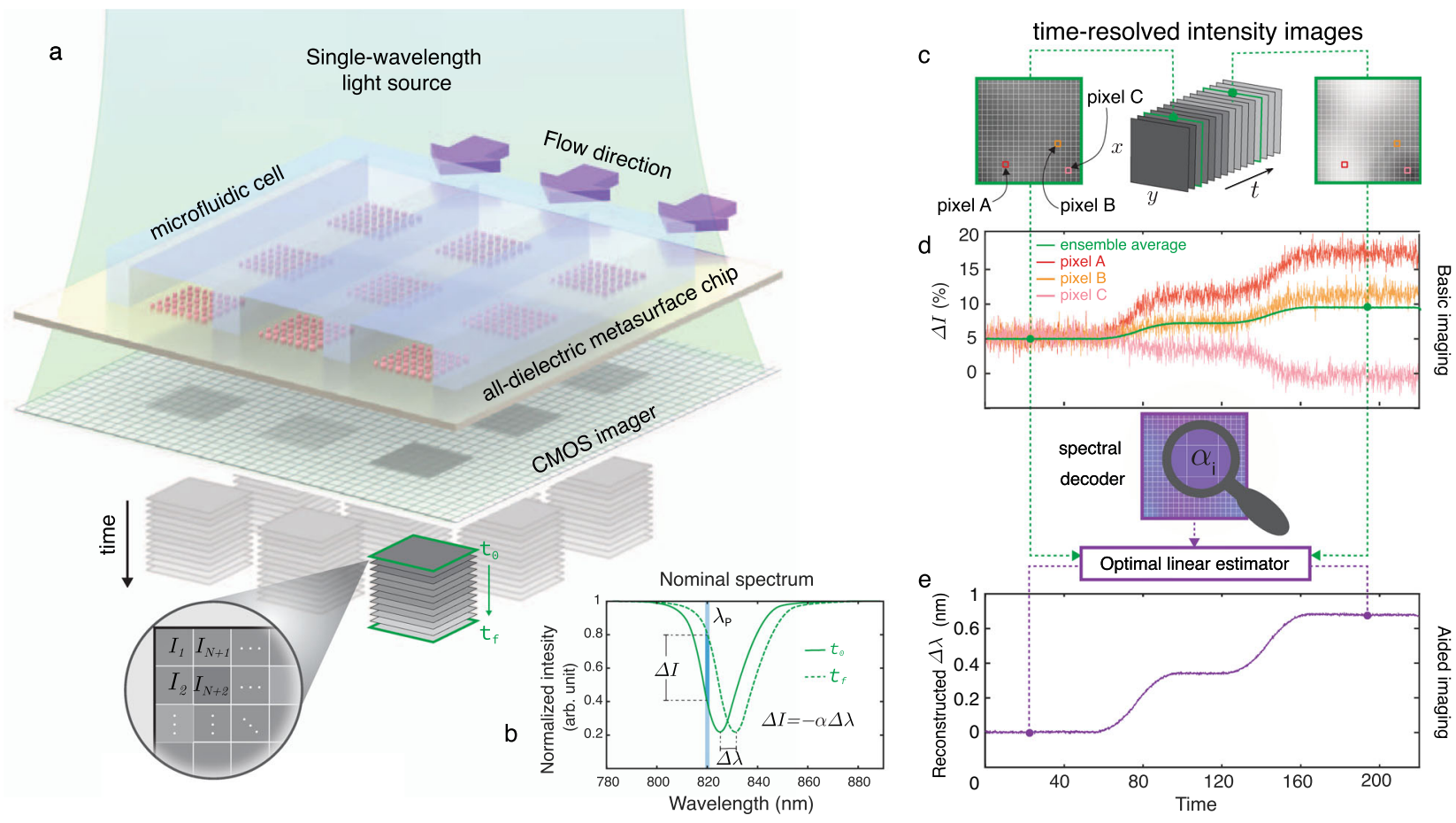

Fig. 1 Principles of the single-wavelength algorithm-aided imaging biosensor with spectral shift reconstruction and its advantages over the basic imaging approach that merely relies on the intensity change. a Sketch of a real-time in-flow imaging platform showing a 2D microarray of all-dielectric sensors integrated with a microfluidic cell consisting of three independent flow channels. The metasurface chip is illuminated with a single-wavelength light source and imaged with a large-area CMOS camera to acquire intensity maps of the sensors in pixel resolution $\left(I_{1}, I_{2}, \ldots, I_{N+1}, I_{N+2}, \ldots\right)$. b Biomarker binding induces a red-shift in the transmission spectrum $(\Delta \lambda)$, which can be detected by tracking the resonance wavelength or by the intensity change $(\Delta /)$ at a fixed probing wavelength $\left(\lambda_{\mathrm{p}}\right) . \Delta /$ can be approximated as linearly proportional to $\Delta \lambda$ with a constant of $\alpha$, which is the slope of the transmission spectrum in the linear region near $\lambda_{p}$. c Pictorial representation of time-resolved single-wavelength intensity images. $\mathbf{d}$ The illustration represents the shortcomings of the basic imaging approach. Time-resolved intensity data from single pixels ( $A, B$, and $C$ ) from the sensor area give contradicting responses with noisy signals (red, orange, and pink curves) due to the nonuniformities. Ensemble averaging of the responses from multiple pixels can help to decrease the noise, although it may reduce the final signal, as depicted with the green curve. e The illustration represents the implementation and the advantages of our aided imaging approach. By leveraging the optimal linear estimator algorithm and the spectral decoder that incorporates the 2D map of the slope values, timeresolved single-wavelength intensity images are used to reconstruct robust spectral shift $(\Delta \lambda)$ information dynamically (purple curve).

quantification of extracellular vesicles binding from solutions with as low as $1.23 \times 10^{8}$ particles $/ \mathrm{mL}$, the equivalent of 204 femtomolar. By fusing the simplicity of intensity-based large-area and real-time imaging detection with the robustness of spectroscopic systems, our technique paves the way for reliable and ultrasensitive POC devices powered by temporally and spatially resolved biosensing spectral shift information.

\section{Results}

The experimental platform. Our all-dielectric biosensor, comprising a $2 \mathrm{D}$ microarray of metasurfaces, is assembled to a microfluidic unit containing three independent and simultaneously accessible flow channels made of Polydimethylsiloxane (PDMS). The optofluidic chip is illuminated with a singlewavelength light beam that excites the resonators. A CMOS camera acquires $2 \mathrm{D}$ images of the whole field of view at fixed time intervals to provide time-resolved intensity information from millions of CMOS pixels (Fig. 1a). Figure 1b shows the red-shift $(\Delta \lambda)$ of the spectral response and its corresponding intensity change $(\Delta \mathrm{I})$ at a fixed probing wavelength $\left(\lambda_{\mathrm{p}}\right)$ due to the binding of the analytes for each pixel. In contrast to spectral interrogation (i.e., measuring directly $\Delta \lambda$ ), basic imaging approaches only rely on the detection of the intensity changes. However, this makes basic imaging vulnerable to the noise factors such as the nonuniform optical response of the resonators over the sensor surface due to the fabrication imperfections and the inadequate choice of the illumination source (i.e., $\lambda_{p}$ ). Figure $1 \mathrm{~d}$ schematically illustrates the problems of the real-time intensity change data extracted from time-resolved images at different pixel locations. The signal coming from a single-pixel is not only noisy, but also contradicts the signals coming from the adjacent pixels (red, orange, and pink curves). Traditional practice to mitigate the noise is to ensemble average the signals from multiple pixels. However, this practice tends to reduce the final signal (green curve) and lowers the sensitivity for operation at low analyte concentrations. To address these shortcomings, we utilized an imaging method relying on the robust spectral shift $(\Delta \lambda)$ information that is extracted without requiring bulky and expensive spectroscopy instrumentation. By using an optimal linear estimation algorithm and a spectral decoder that is unique to each metasurface sensor, we reconstruct spectral shift data from timeresolved single-wavelength intensity images dynamically. This algorithm-aided imaging approach can increase the signal to noise ratio (SNR) and, consequently, improve the limit of detection (LOD) by unveiling the signals coming from a highly diluted analyte, which is otherwise unresolvable by conventional ensemble averaging methods (Fig. 1e).

All-dielectric metasurfaces. To engineer our all-dielectric metasurfaces for biosensing, we developed the universal theory of quasi-BICs in broken-symmetry dimer-type metasurfaces (see Supplementary Information Note 2). The advantage of such 
a

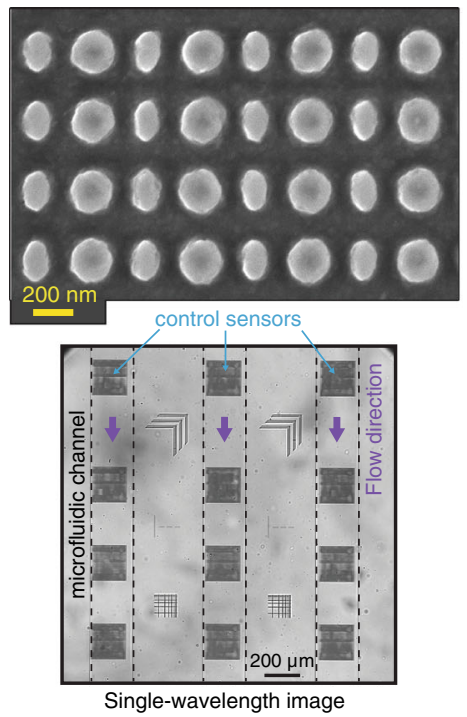

b

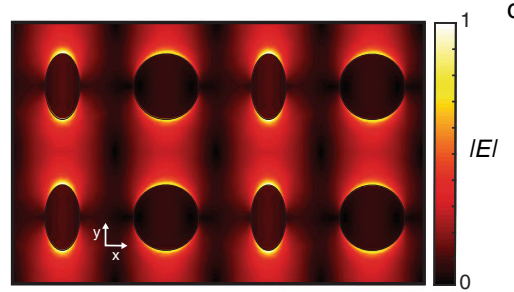

e

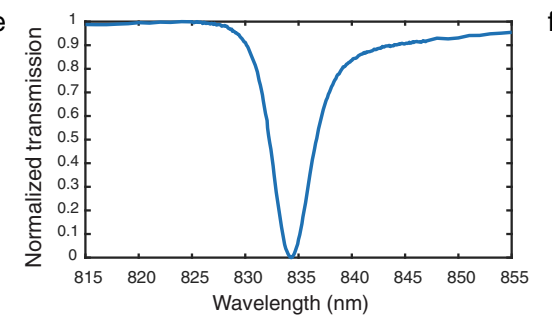

c

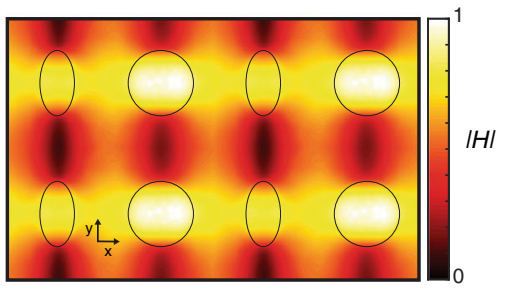

$\mid H I$

f

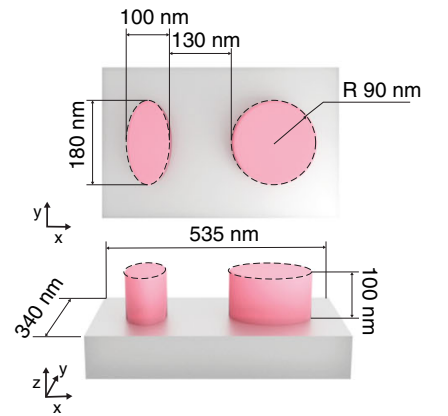

Fig. 2 Diatomic dielectric metasurface supporting high quality factor quasi-BIC resonances. a Scanning electron microscopy (SEM) image of the

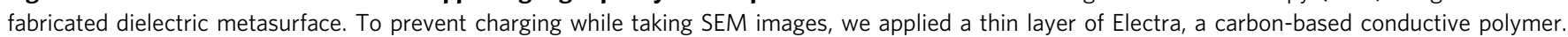

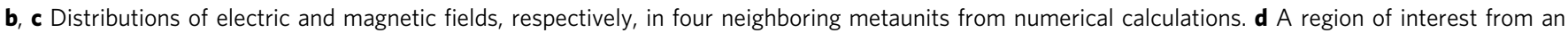
intensity image obtained at the resonance wavelength of the integrated optofluidic chip showing $3 \times 4$ all-dielectric metasurface sensors and borders of the three Polydimethylsiloxane (PDMS) flow channels. e Experimentally acquired transmission spectrum of the fabricated dielectric metasurfaces in ypolarization. $\mathbf{f}$ Top and side-views schematic of the metaunit composed of an elliptical (long and short axis $180 \mathrm{~nm}$ and $100 \mathrm{~nm}$ respectively) and a cylindrical (90 $\mathrm{nm}$ in radius) a-Si resonators.

diatomic metasurfaces is a higher number of degrees of freedom to achieve better precision in tuning the accessible enhanced near-fields and the quasi-BIC Q. The meta-molecule consists of a circular and an elliptic disk-this allows breaking the in-plane inversion symmetry (Fig. 2f). The asymmetry is defined as the degree of ellipticity of one of the meta-atoms, which is proportional to the difference between the ellipse long and short axes. We observed that controlling the asymmetry along one axis improves the tolerance to the fabrication imperfection than the alternative methods such as the one used in our previous work ${ }^{38}$. Nonzero ellipticity is a perturbation of a circular disk and induces leakage of radiation out of the metasurface plane at the BIC conditions (Supplementary Fig. 3). The BIC transforms into a quasi-BIC with finite radiative losses controlled by the asymmetry of the meta-molecule. Due to the reciprocity, the quasi-BIC manifests itself as a narrow resonant feature in the transmission spectrum for y-polarized incident light (Supplementary Fig. 3). Our design supports highly accessible enhanced electric and magnetic fields in the external surface of the dielectric nanoresonator (Fig. 2b, c). The local electric field enhancement factors can reach as high as almost 5000 times (Supplementary Fig. 4), and the enhancement of the electric field averaged over a volume of a 5-nm thick layer in the vicinity of the nanoresonators in an aqueous media is calculated to be 12.4 times. Overall, this design gives rise to a considerable intensification of the optical interaction with the analytes and makes the platform suitable for sensing applications ${ }^{46-48}$.

Figure $2 \mathrm{a}$ shows a scanning electron microscope (SEM) image of the resonators fabricated by nanostructuring a 100 -nm-thick amorphous silicon (a-Si) on top of a fused silica substrate using a top-down fabrication method with CMOS compatible techniques. Silicon is chosen as the constituent material due to its biocompatibility, well-established surface biofunctionalization methods, and access to mature CMOS fabrication facilities. Also, silicon benefits from desirable optical properties, including a high refractive index and relatively low loss in the near-IR range below $850 \mathrm{~nm}$. The metasurfaces operate in the aqueous media with their resonance in the visible and near-IR spectral range $(600 \mathrm{~nm}$ to $850 \mathrm{~nm}$ ). Working in this range offers several unique advantages, including the wide availability of high-performance and cost-effective silicon-based CMOS and CCD cameras. For fabrication, we used the design with geometrical parameters shown in Fig. 2f, which corresponds to the ellipticity of 0.44 . As mentioned above, the metasurface design is tolerant to fabrication imperfections due to the chosen symmetry-breaking strategy. The fabricated resonators were characterized to have $<2 \%$ size deviations over the sensor area. In turn, a good homogeneity is observed in the optical response of the resonators (see supplementary information note 4).

Our metasurface chip comprises twelve $250 \mu \mathrm{m}$ by $250 \mu \mathrm{m}$ sensors in a $3 \times 4$ microarray format. The optofluidics incorporates three independent microfluidic channels, which allow the sensors to function in aqueous media for in-flow measurements and preserve analyte biofunctionality. The field of view (FOV) of the setup is $>7 \mathrm{~mm}^{2}$; thus, we can acquire the images of all of the twelve sensors on a chip in a single shot. Figure $2 \mathrm{~d}$ shows a subset of an intensity image obtained at the resonance wavelength of the all-dielectric metasurface. The sensors, as well as the borders of the three PDMS fluidic channels, are visible at high contrast. The cross patterns in between the microfluidic channels are alignment and focus monitoring marks, which are used during the subsequent data processing of the time-resolved intensity images. Our aided imaging approach, based on an optimal linear estimation algorithm and a spectral decoder, allows for precise reconstruction of the spectral shift over large areas from the acquired images. The spatially resolved reconstructed shift data from a large FOV has a compelling advantage over the traditional spectrometers by allowing for a concurrent control test and multiplexing to detect a panel of biomarkers in parallel. Figure $2 \mathrm{e}$ shows the experimentally recorded transmission spectrum of the 

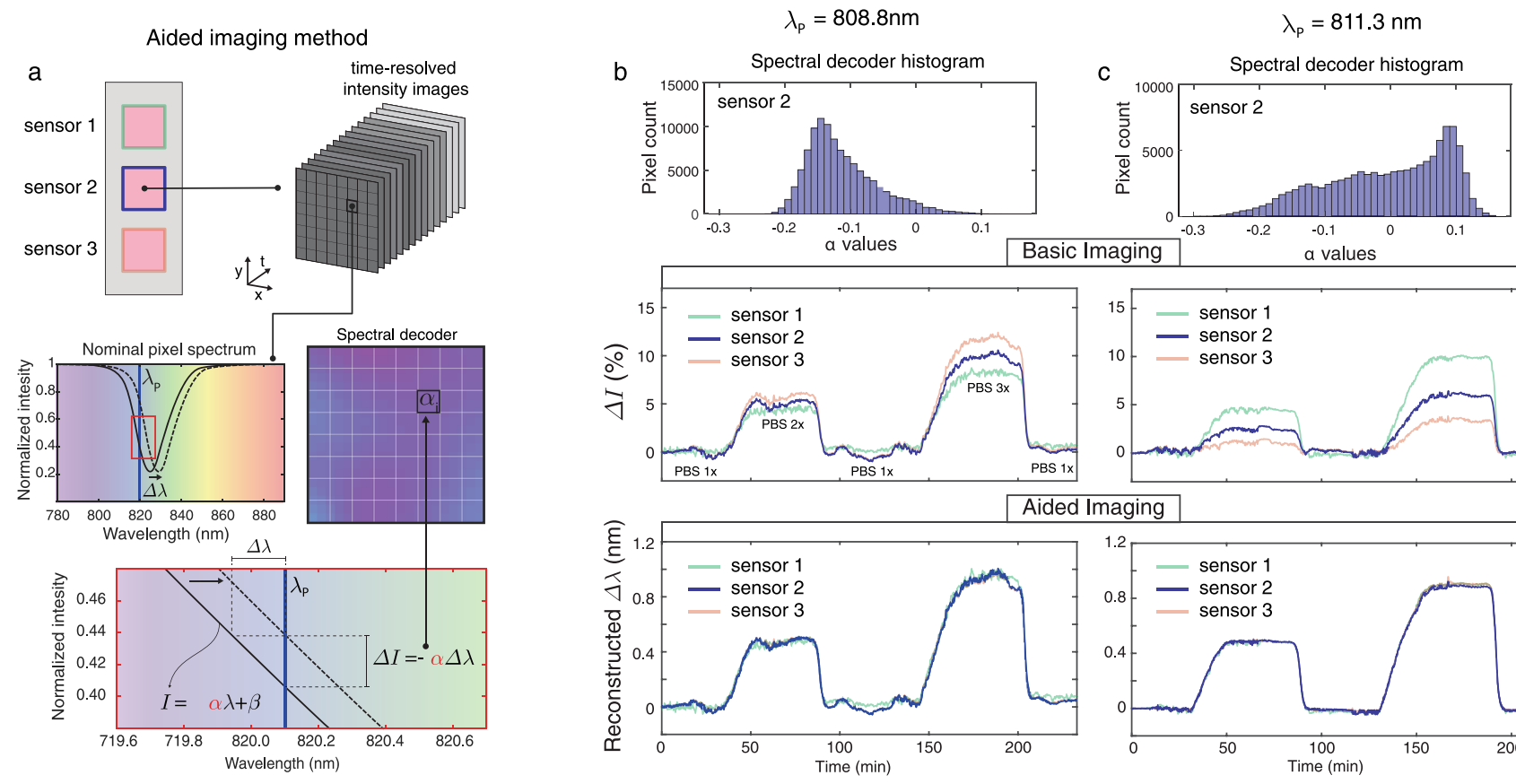

Fig. 3 Implementation of the algorithm-aided imaging technique with reconstructed spectral shift information. a Time-resolved intensity data is fed to the optimal linear estimation algorithm with a spectral decoder, which is the two-dimensional (2D) map of the slope ( $\alpha$ ) of the transmission spectrum for each CMOS pixel at the probing wavelength $\left(\lambda_{\mathrm{p}}\right)$. b At $\lambda_{\mathrm{p}}=808.8 \mathrm{~nm}$, the histogram of $\alpha$ values from a single sensor is populated mostly with negative values because, for the majority of the pixels, the resonance is probed at the left flank of the transmission dip (top panel). This case represents a sensor having a relatively homogenous optical response across its surface. For experimental bulk sensing, under a constant running of phosphate buffer saline (PBS) $1 \mathrm{x}(n=1.33441)$, three sensors are exposed to a varying medium with PBS $2 x(n=1.33584)$ and PBS $3 x(n=1.33731)$ and their ensemble-averaged intensity changes $\left(\Delta /\right.$, middle panel) and reconstructed spectral shifts $\left(\Delta \lambda\right.$, bottom panel) are presented as a function of time. $\mathbf{c}$ At $\lambda_{\mathrm{p}}=811.3 \mathrm{~nm}$, the histogram of the same sensor has a similar number of positive and negative $\alpha$ values (top panel). This case represents a sensor having an inhomogenous optical response. For the same bulk sensing experiment used in Fig. $3 b$, the ensemble-averaged $\Delta /$ response of different sensors shows significantly varying signals (middle panel). In contrast, the reconstructed spectral shift data obtained by aided imaging (bottom panel) is consistent between the three sensors.

engineered sensor. The optical characterization of 12 sensors, which are sampled with more than 950,000 CMOS pixels in the field of view, shows that the full-width at half-maximum (FWHM) of the resonance is as low as $2.3 \mathrm{~nm}$ and on average $4.57 \mathrm{~nm}$ with a standard deviation of $0.47 \mathrm{~nm}$. Consequently, a $Q$ as high as 250 and on average 178.6 with a standard deviation of 15.8 was recorded experimentally. Owing to the high- $Q$ resonances and highly accessible enhanced near-fields, we achieved an experimental bulk refractive index sensitivity of $305 \mathrm{~nm} / \mathrm{RIU}$ and a figure-of-merit (defined as sensitivity divided by FWHM) of 68 1/RIU.

Aided imaging method. Figure 3 elucidates the gained robustness and tolerance of the biosensor when the aided imaging method is implemented compared to the basic imaging. To reconstruct spectral shift $(\Delta \lambda)$, we fed the time-resolved intensity data to an optimal linear estimation algorithm that uses a "spectral decoder" and the recorded intensity change of each CMOS pixel to compute the best estimate of $\Delta \lambda$ for the entire sensor. The spectral decoder of a sensor is built up by gathering the values of pixel-wise slopes $(\alpha)$ across the sensor area. To do so, we linearize the spectrum associated with each pixel around the probing wavelength $\lambda_{p}$ and compute its slope (see Fig. 3a). This can be simply obtained by recording two images using narrowband illuminations: one at $\lambda_{p}$ and a second one at $\lambda_{p}+\delta$, for a small value of $\delta$. This decoder provides information about the weight of the contribution of each pixel to the spectral shift so that the more sensitive ones have a higher influence on the estimation of $\Delta \lambda$. In this work, the spectral decoder was computed using a hyperspectral imaging system where the sensor was imaged at wavelengths with a small spectral increment of $0.1 \mathrm{~nm}$, and the extracted $\alpha$ values are mapped as a $2 \mathrm{D}$ matrix. To experimentally demonstrate the advantages of our aided imaging method over basic imaging, we studied two cases of uniform and non-uniform optical responses over the sensor area at different probing wavelengths. The experiments involved recording simultaneously time-resolved intensity images of three different sensors assembled in a microfluidic channel and comparing their responses under the same condition. This is achieved by flowing aqueous solutions with varying refractive indices across the sensors with consecutive injections of phosphate buffer saline (PBS) at different concentrations. Figure $3 b, c$ show the responses of the three sensors at the illumination wavelengths of $808.8 \mathrm{~nm}$ and $811.3 \mathrm{~nm}$, respectively. In the first case shown in Fig. $3 \mathrm{~b}$ (top panel), the spectral decoder histogram of a representative sensor (sensor 2) is populated dominantly with negative values, indicating that the probing wavelength intersects the spectra of most pixels on the left flank of the resonance dip. Even with relatively uniform distributions, the responses of the three sensors obtained with basic imaging differ in the intensity change sensorgram (Fig. 3b, middle panel) compared to the aided imaging method (Fig. 3b, bottom panel). If the nonuniformity increases, the performance of the basic imaging deteriorates even more, as shown with the second case in Fig. 3c. In this figure (top panel), the spectral decoder histogram has a similar number of positive and negative values. Consequently, in the ensemble averaging with the absolute intensity change, the pixels with oppositive-valued slopes work against one another and result in significant variations between the three responses, leading to poor sensing performance (Fig. $3 c$, middle panel). In contrast, when the spectral decoder is used in the context of the optimal estimator, we obtained almost identical responses from all three sensors 
(Fig. 3c, bottom panel). The drastically improved performance illustrates that our technique is robust to the inhomogeneity of sensor response that is caused by, for instance, small fabrication imperfections or the improper choice of probing wavelength. This is a significant step toward inexpensive POC instruments for on-site use in resource-limited settings since it eliminates the need for the elusive perfect fabrication of the sensors as well as allows for flexibility in the choice of probing wavelengths. Moreover, our technique provides the reconstruction of the spectral shift without the need for a bulky and expensive spectrometer.

Biosensing experiments. To demonstrate the biosensing functionality of our aided imaging method, we performed real-time in-flow detection of nanoparticles (NPs), as well as biological extracellular vesicles (EVs) secreted from cancer cells with an immunoassay. Initially, biotinylated silica nanoparticles $(100 \mathrm{~nm}$ diameter) were used as the mimics of bioparticles (i.e., EVs and viruses) for the characterization of the sensors ${ }^{49,50}$. Each microfluidic flow channel interacts with four dielectric metasurface sensors (see Fig. 2d). The first sensor, which is blocked with bovine serum albumin (BSA), serves as a control sensor. The successive three sensors (detection sensors) are locally functionalized with streptavidin protein to target the biotin molecules on the silica nanoparticles (Supplementary Fig. 6). A steady flow of phosphate buffer saline (PBS) 1X delivers a sequence of different dilutions of the NP solution to the sensors, which are continuously illuminated with a linearly polarized single-wavelength light and imaged in time intervals of $14 \mathrm{~s}$ by a CMOS camera. The reported $\Delta \lambda$ in the $y$-axis is the reconstructed shift observed on the detection sensors from which the reconstructed shift of the control sensor is subtracted to account for any non-specific bindings.

The calibration curve extracted from the reconstructed shift induced by the binding events of the silica nanoparticles on the alldielectric metasurfaces is shown in Fig. 4a. The error bars in the figure are representing the standard deviation of $\Delta \lambda$ over time (sensorgram) from each sensor over a time-interval consisting of 81 consecutive images after the analyte binding reaches the equilibrium state. The relative standard deviation for the reproducibility of $\Delta \lambda$ in the working range of sensors is observed to be between $0.33 \%$ and $3.97 \%$. LOD is the smallest concentration that can be detected with reasonable certainty for a biosensor ${ }^{51}$. The dashed red line in Fig. $4 \mathrm{a}$ shows the three times the averaged standard deviation, which was calculated by averaging the standard deviation of the sensorgram signals at equilibrium states over at least four time-intervals, each consisting of 81 consecutive images. The LOD of $1.2 \times 10^{8}$ nanoparticles/mL, the equivalent of 199 femtomolar that corresponds to $126 \mathrm{ng} / \mathrm{mL}$, was extracted from the intersection of the dashed red line and the calibration curve. The lowest detected quantity of $1.9 \times 10^{8}$ nanoparticles/mL or 315 femtomolar, corresponding to $200 \mathrm{ng} / \mathrm{mL}$, was measured and further investigated with SEM images revealing the equivalent detection of 0.41 nanoparticles $/ \mu \mathrm{m}^{2}$ averaged over 78 SEM images and the total area of $687 \mu \mathrm{m}^{2}$ at randomly chosen locations on the sensors with the standard deviation of 0.26 nanoparticles/ $\mu \mathrm{m}^{2}$ and 0.34 nanoparticles/ $\mu \mathrm{m}^{2}$ as the median. The insets of Fig. 4a shows representative SEM images from the solutions with $1.9 \times 10^{8}$ nanoparticles $/ \mathrm{mL}$ and $9.5 \times 10^{12}$ nanoparticles $/ \mathrm{mL}$ from left to right, respectively.

To demonstrate the competence of the proposed method with a biorecognition assay, we detected and quantified EVs purified from the cell culture supernatant of 4T1 mouse breast cancer cells $^{45}$. EVs, which include exosomes and microvesicles, are nanoscale bioactive membrane particles secreted by virtually all types of cells ${ }^{52}$. There is strong evidence that EVs derived from breast cancer cells influence tumor progression ${ }^{45}$. EVs carry molecular information from parental cells; therefore, they can be considered as biomarkers for early-stage cancer detection and for minimally or non-invasive diagnostics ${ }^{44}$. A transmission electron microscopy (TEM) image of the EVs is shown in Fig. 4d, and their purification process and quantification are briefly explained in the methods section. EVs are highly enriched in tetraspanins ${ }^{52}$. The tetraspanins superfamily is a family of proteins with at least 33 distinct members in mammals; among them, CD9 is the beststudied transmembrane protein and a versatile marker that has been used for EV subtypes, including exosomes ${ }^{53,54}$. In order to capture the vesicles, we targeted CD9 and immobilized the corresponding biotinylated KMC8 anti-CD9 monoclonal antibody on the detection sensors functionalized with streptavidin. The control sensor was blocked with BSA (Fig. 4b). As in the nanoparticle experiment, a pressure pump maintains the constant buffer (PBS 1x) flow in the microfluidic channel, and a syringe pump provides successive injections of EV samples in various concentrations. Figure $4 \mathrm{c}$ shows the calibration curve that uses the reconstructed shifts derived from single-wavelength real-time in-flow experiments to detect EVs from 4T1 mouse breast cancer cells. The error bars in the figure are calculated in the same manner as in Fig. 4a. In this case, the relative standard deviation for reproducibility varies between $0.50 \%$ and $8.11 \%$. Our sensors have a limit of detection of $8 \times 10^{7} \mathrm{EV} / \mathrm{mL}$ or 133 femtomolar, according to the intersection of the three times the averaged standard deviation (the dashed red line in Fig. 4c) with the calibration curve, and the lowest detected quantity was measured as $1.23 \times 10^{8} \mathrm{EV} / \mathrm{mL}$ or 204 femtomolar. The $\mathrm{EV}$ protein content at these concentration values corresponds to $267 \mathrm{ng} / \mathrm{mL}$ and 423 $\mathrm{ng} / \mathrm{mL}$, respectively ${ }^{45}$. While typically correlating with the tumor burden, the average values of cancer-related EVs range between $10^{9}$ and $10^{10}$ per $\mathrm{mL}$ of human plasma ${ }^{55-57}$. With a LOD of $8 \times$ $10^{7} \mathrm{EV} / \mathrm{mL}$ and about five orders of magnitudes dynamic range, our results indicate that the performance of the proposed sensor is clinically relevant for the detection of cancer-related EVs.

\section{Discussion}

We demonstrated a label-free nanophotonic biosensor, which leverages an aided imaging approach based on novel data processing and an optofluidic chip comprising of all-dielectric metasurfaces. Our method enables the extraction of spectral shift information from single-wavelength intensity images at high accuracy while preserving temporal and spatial resolution. Importantly, it is based on simple imaging optics with a fixedwavelength illumination and a CMOS camera to collect data over a large field of view in real-time. Compared to the basic intensity imaging readers that merely rely on intensity change, our approach aided by optimal linear estimation algorithm provides robustness to the spectral inhomogeneities across the sensor surface that can originate from fabrication imperfections and inadequate operation wavelength. The current device, which images over $7 \mathrm{~mm}^{2}$ field-of-view, can be used to perform simultaneous measurement from hundreds of individual sensors in a microarray format. Hence our approach is suitable for multiplexed detection of a panel of biomarkers in-real time for highthroughput monitoring. Our diatomic all-dielectric metasurface, emanated from quasi-BIC modes, enables high-performance sensing by supporting high $Q$ resonances with accessible nearfields. Furthermore, its unique symmetry-breaking design makes it more tolerant of nanofabrication. Using CMOS-compatible techniques, we fabricated highly uniform silicon metasurfaces operating below $850 \mathrm{~nm}$ in aqueous media and integrated with microfluidics for in-flow measurements. As a proof-of-concept, we applied the new optofluidic device for real-time detection of breast cancer derived EVs, and by functionalization of the 
a

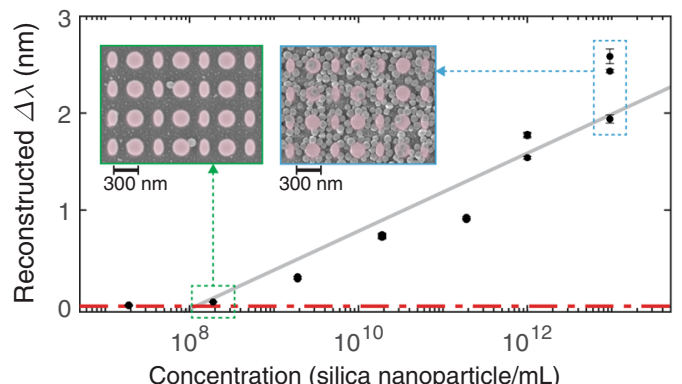

$\mathrm{C}$

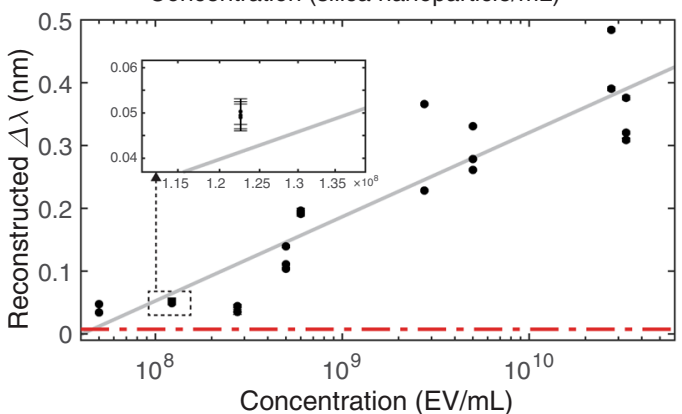

b

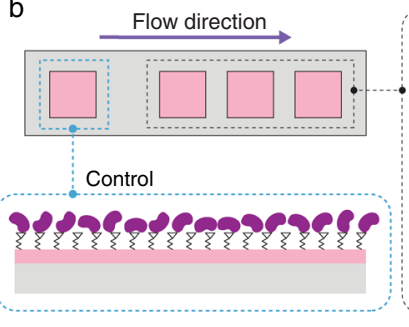

そEpoxy silane Biotin BSA

d

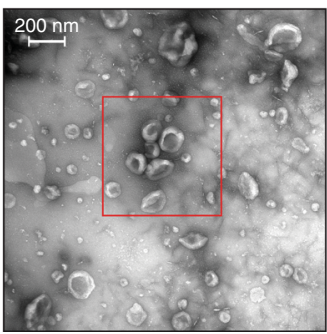

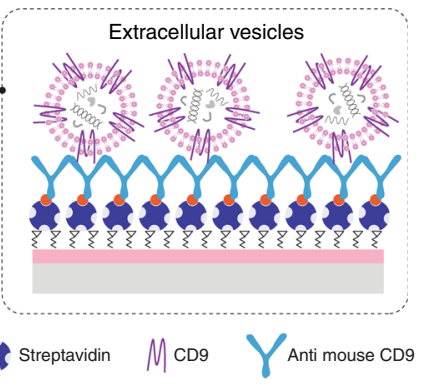

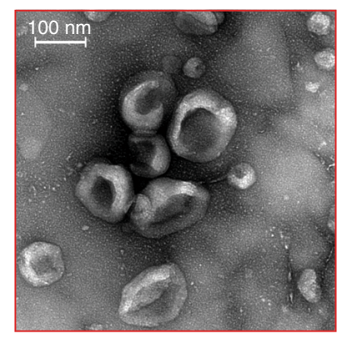

Fig. 4 Biosensing using reconstructed spectral shift based on aided imaging method. a The reconstructed spectral shift ( $\Delta \lambda$ ) calibration curve of biotinylated silica nanoparticles (100 nm diameter). The insets are scanning electron microscopy images of the sensors with bound silica nanoparticles on resonators after introducing suspensions with $1.9 \times 10^{8}$ nanoparticles $/ \mathrm{mL}$ and $9.5 \times 10^{12}$ nanoparticles/mL from left to right, respectively. The represented data points in the calibration curves $(\mathbf{a}, \mathbf{c})$ are the mean $\Delta \lambda$ over time from each sensor over a time-interval consisting of 81 consecutive images after the analyte binding reaches the equilibrium state, and the error bars indicate the standard deviations. The reconstructed $\Delta \lambda$ data points are obtained from seven dilutions of silica nanoparticle solutions over three independent sensors to achieve 21 data points on the calibration curve. The reconstructed $\Delta \lambda$ data from the detection sensors are corrected to the non-specific bindings on the control sensor. Some data points are overlapped due to the good agreement between the sensor performance. $\mathbf{b}$ Biorecognition assay to detect extracellular vesicles (EVs) on the detection sensors and on the control sensor blocked with bovine serum albumin (BSA) to account for non-specific binding is shown on top. c The reconstructed spectral shift calibration curve of EVs. The inset is the same plot with a magnified $x$-axis to better resolve the small error bars for an exemplary data point. The reconstructed shift data points from the detection sensors are corrected to the non-specific bindings on the control sensor. The represented data points are collected from nine different concentrations of EV solutions diluted from three independent batches measured on eight independent sensors to yield 24 data points on the calibration curve. Some data points are overlapped due to the good agreement between the sensors. d Transmission electron microscopy images of EVs from the cell culture supernatant of 4 T1 mouse breast cancer cells can be seen at the bottom; the magnifications are $\times 23,000$ and $\times 30,000$ from left to right, respectively.

metasurfaces with specific antibodies, we were able to report the LOD of down to 133 femtomolar.

As an outlook, the flexibility of the detection principle can enable the expansion into various different application areas. For instance, with proper surface functionalization, we can selectively target other bioparticles such as intact viruses for infectious disease applications and smaller biomolecules, including proteins, antibodies, and nucleic acids. We anticipate wafer-scale and highthroughput fabrication techniques to eliminate the dependence on high-resolution e-beam lithography. This can drastically reduce the cost and enable disposable biochips customized as consumables for detecting different analytes. We foresee device integration in miniaturized biosensors by using small-scale light sources with tailored bandwidth and low-cost on-chip imagers. The use of large-area imaging data gives the opportunity of implementing other advanced computation techniques (i.e., artificial intelligence methods) towards smarter sensing. By eliminating the need for bulky and expensive instrumentation, the spectrometer-less device architecture can lead to affordable, compact, easy-to-use, and reliable point-of-care systems for disease diagnostics, personalized medicine, and democratization of health care in resource-limited settings.

\section{Methods}

All-dielectric metasurface fabrication. A thin layer of amorphous silicon (a-Si) with $100 \mathrm{~nm}$ thickness was deposited using low-pressure chemical vapor deposition (LPCVD) on a clean bare fused-silica wafer. The wafer was patterned using $100 \mathrm{KeV}$ electron-beam lithography (EBL). First, the wafers were cleaned with radio corporation of America (RCA) cleaning solution and oxygen plasma. A positive-tone resist, poly(methyl methacrylate) in a double layer configuration (PMMA $495 \mathrm{~K}$ bottom and $950 \mathrm{~K}$ top) was spin-coated and baked for $5 \mathrm{~min}$ at $180^{\circ} \mathrm{C}$ to yield the total thickness of $160 \mathrm{~nm}$. PMMA double-layer configuration is crucial to make undercut structures to facilitate lift-off. A 10 -nm gold layer is sputtered on the PMMA layer to ensure conductivity during electron-beam writing. After EBL exposure, the gold layer was etched away in $\mathrm{KI}+\mathrm{I}_{2}$ solution, and the wafer was developed with a mixture of Methyl isobutyl ketone (MIBK) and IPA (Isopropyl alcohol) in 1-3 ratios. To make a hard mask, a 30-nm-thick aluminum layer was deposited using an electron-beam evaporator followed by dicing the wafer into $1.5 \mathrm{~cm} \times 1.5 \mathrm{~cm}$ chips. Metal lift-off was done by soaking the chips in a resist-remover bath at $70{ }^{\circ} \mathrm{C}$ for $6 \mathrm{~h}$. The fabrication process concludes with transferring the nanostructures to the a-Si layer using fluorine-based deep reactive ion etching, and finally, hard mask removal in aluminum etch solution.

Optical characterization setup. All-dielectric metasurfaces were optically characterized using a hyperspectral (HSI) platform. The HSI system consists of a supercontinuum laser (SuperK EXTREME EXR-15, NKT photonics) coupled to a tunable filter (LLTF, NKT photonics) integrated with an inverted microscope (Eclipse-Ti, Nikon). The collimated linearly polarized laser line $(1.75 \mathrm{~nm}$ bandwidth at $700 \mathrm{~nm})$ light was transmitted from the metasurface and collected with a $\times 10$ objective, conveyed through a collinear analyzer and imaged with a CMOS camera (DS Qi 2, Nikon). To extract the transmission spectrum of all-dielectric metasurfaces with pixel size spatial resolution over a large field of view, a hyperspectral data cube was acquired by continuously imaging the chip synchronized with the sweep of the illumination source wavelength. The hyperspectral data cube of a chip was normalized to the hyperspectral data cube of the light source. The normalized intensity profile of each pixel over the wavelength sweep yields the transmission spectra of that pixel. The devices were connected and controlled with a centralized Matlab code.

Single -wavelength imaging-based real-time biosensing measurements. The optical setup for the real-time in-flow measurements was the same as the optical characterization setup. A biofunctionalized chip was assembled to a microfluidic 
structure and placed on a microscope stage. The microfluidic system consists of a pressure pump (Elveflow, OB1) to maintain the constant buffer (PBS 1x) flow in the microfluidic channel, a syringe pump (Advanced MicroFluidics, LSPone) for analyte injection with a flowmeter (Elveflow, FS3) and tubing. Our data was collected from the net sample volume of $150 \mu \mathrm{L}$, which is more than one order of magnitude smaller than a conventional blood test. This volume can be further decreased to few tens of $\mu \mathrm{Ls}$ if in-flow measurements and real-time data are not needed, and the measurements are performed under static conditions. Image acquisition in 14-second intervals and data pre-processing were handled with the centralized Matlab code assisted by a macro from NIS Nikon software. The preprocessing module was capable of providing live feedback by plotting the reconstructed spectral shift over time.

Full-wave numerical analysis. Numerical analysis of the all-dielectric metasurfaces was performed using a commercially available finite-element frequencydomain solver (CST Microwave Studio 2018).

Microfluidic structure fabrication. The microfluidic structures were made by soft lithography using a silicon master mold to achieve three $300 \mu \mathrm{m}$ wide individual flow channels that are simultaneously accessible. The master mold was made with photolithography techniques to achieve the patterns of the final microfluidic channel. A film of positive-tone resist (AZ 1512, $1.3 \mu \mathrm{m})$ was coated and patterned on a silicon wafer using an automatic coater and developer (EVG 150, EV Group) and direct laser writer (MLA 150, Heidelberg). The patterned photoresist was used as an etching mask for fluorine-based silicon dry etch in a deep reactive ion etching (DRIE) tool (AMS 200, Adixen) to make $25 \mu \mathrm{m}$ thick patterns. Finally, the photoresist is removed with oxygen plasma cleaning. One mold can be used repeatedly if the surface is protected from permanent adhesion of PDMS by silanization. Silanization was done using TriMethylChloroSilane (TMCS) in a desiccator under vacuum to make TMCS evaporate and form a passivation layer on the mold surface. The base and crosslinker precursors were mixed in a 10 to 1 ratio to make PDMS. The precursor mixture was degassed in a desiccator and poured into the mold. After another degassing step to ensure removing all the bubbles from the mold's surface, it was baked at $80^{\circ} \mathrm{C}$ for $4 \mathrm{~h}$. Finally, the inverted replica of the mold in PDMS was peeled off from the mold, and each pattern was cut, punched, and bound to its complementary part with oxygen plasma.

Surface chemistry. Covalent surface chemistry based on (3-glycidoxypropyl) trimethoxysilane (3-GPS) (Sigma-Aldrich) monolayer was used to immobilize the capture molecules on the all-dielectric metasurface. The silane end bonds to the silicon oxide and covers the all-dielectric metasurface with a uniform 3-GPS monolayer. The epoxide functional group of 3-GPS binds to the amine group of the capture molecules. First, the chips were cleaned in RCA solution at $50{ }^{\circ} \mathrm{C}$ for $30 \mathrm{~min}$. The clean chips were incubated in the $3-\mathrm{GPS}$ solution in toluene $(1 \% \mathrm{vol} /$ vol) for $20 \mathrm{~min}$ and rinsed in fresh toluene to remove the unreacted 3-GPS molecules and dried. The chips were baked for $30 \mathrm{~min}$ at $120^{\circ} \mathrm{C}$ and were stored under a vacuum to be used within a week.

Biotinylated silica nanoparticles biorecognition assay. To specifically target the Biotinylated silica nanoparticles (Creative Diagnostics) of $100 \mathrm{~nm}$ diameter, streptavidin was covalently immobilized on the detection sensors as a capture molecule, and BSA (Sigma-Aldrich) was immobilized on the control sensor. Epoxy-silane chemistry described above is used for the immobilization of a monolayer of Streptavidin and BSA molecules on the detection and control sensors, respectively. 40 droplets $(480 \mathrm{pL}$ per droplet) of Streptavidin (Thermo Scientific, $1 \mathrm{mg} / \mathrm{mL}$ ) and BSA (1\% wt/vol) in PBS (Sigma-Aldrich) $1 \mathrm{x}$ buffer were spotted on the sensors with a low-volume liquid dispenser (sciFLEXARRAYER S3, Scienion). The spotted chip is incubated for 16 hours at $4{ }^{\circ} \mathrm{C}$

EV biorecognition assay. All-dielectric metasurfaces were initially treated with epoxy-silane chemistry and functionalized with a monolayer of streptavidin on the detection sensors and BSA on the control sensors by spotting technique. Streptavidin and BSA molecules are incubated for $2 \mathrm{~h}$ at room temperature and rinsed consecutively with PBS $1 \mathrm{x}$ and Mili-Q water, and then dried. In all, 40 droplets ( $480 \mathrm{pL}$ per droplet) of a biotinylated capturing molecule, KMC8 monoclonal antibody (Biotin Rat Anti-Mouse CD9, BD biosciences, $0.5 \mathrm{mg} / \mathrm{mL}$ ) in PBS 1x were spotted on detection sensors and BSA on the control sensors. The spotted chip is incubated for $16 \mathrm{~h}$ at $4^{\circ} \mathrm{C}$.

EV isolation. EVs were purified from media conditioned by $4 \mathrm{~T} 1$ cells. In all, 4T1 cells (mammary adenocarcinoma; from ATCC) were cultured in $15 \mathrm{~cm}$ tissue culture-treated dishes in Iscove's Modified Dulbecco's Medium (IMDM, Sigma) supplemented with $10 \%$ fetal bovine serum (FBS; Gibco), L-glutamine (Amimed), and penicillin/streptomycin (Gibco). Cells at $\sim 40-50 \%$ confluence were moved to a medium containing $5 \% \mathrm{EV}$-depleted $\mathrm{FBS}^{45}$. EVs were isolated from the conditioned medium using sequential ultracentrifugation $(500 \times g$ for $5 \mathrm{~min} ; 2000 \times g$ for $10 \mathrm{~min} ; 4600 \times g$ for $20 \mathrm{~min} ; 134,000 \times g$ for $70 \mathrm{~min})$. The resulting EV pellet was washed in PBS, ultracentrifuged again at $134,000 \times g$ for $70 \mathrm{~min}$, and finally dissolved in PBS, which was obtained by ultracentrifugation of standard FBS at $134,000 \times g$ for $16 \mathrm{~h}$ at $4{ }^{\circ} \mathrm{C}$ followed by filtration through a $0.1-\mathrm{mm}$ vacuum filtration bottle. After 3 days, the conditioned medium was collected and centrifuged at $500 \times g$ for $5 \mathrm{~min}, 2000 \times g$ for $10 \mathrm{~min}$ to remove dead cells and debris, and ultracentrifuged at $4600 \times g$ for $20 \mathrm{~min}$ at $4{ }^{\circ} \mathrm{C}$ to remove large vesicles. The supernatant was then transferred to new tubes and ultracentrifuged at $134,000 \times g$ for $70 \mathrm{~min}$ at $4{ }^{\circ} \mathrm{C}$ to collect small EVs. The EV pellet was then washed in $35 \mathrm{~mL}$ of PBS and ultracentrifuged again at $134,000 \times g$ for $70 \mathrm{~min}$ at $4{ }^{\circ} \mathrm{C}$. All ultracentrifugation steps were performed using a Beckman ultracentrifuge and a SW32Ti rotor. The resulting EV preparation was resuspended in PBS and stored at $-80{ }^{\circ} \mathrm{C}$.

Transmission electron microscopy of purified EVs. Purified EVs $(5 \mu \mathrm{g}$ in $15 \mu \mathrm{L}$ of PBS) were applied to carbon-coated 400 mesh grids (Electron Microscopy Sciences) for $5 \mathrm{~min}$, then washed with PBS and stained with $2 \%$ uranyl acetate (Sigma) for $30 \mathrm{~s}$. Excess stain was removed by touching the very edge of the mesh with a piece of filter paper (Whatman). Grids were then let dry completely prior to acquisition. Images were obtained using a transmission electron microscope device (Tecnai Spirit, FEI Company).

Computation of spectral decoder. Given a probing wavelength $\lambda_{\mathrm{p}}, 11$ images are recorded at wavelengths according to the column vector $\lambda=\left[\lambda_{\mathrm{p}}-0.5, \ldots, \lambda_{\mathrm{p}}, \ldots, \lambda_{\mathrm{p}}\right.$ $+0.5] \mathrm{nm}$. For each pixel $i$, the recorded intensities are normalized according to the intensity value corresponding to $\lambda_{\mathrm{p}}$, resulting in a vector $y^{i}$, where $y^{i}[6]=1$. The slope associated with pixel $i$ is then given by Eq. (1)

$$
\alpha^{i}=\frac{\left(y^{i}-\overline{y^{i}}\right)^{T}\left(\lambda^{i}-\overline{\lambda^{i}}\right)}{\left(\lambda^{i}-{\overline{\lambda^{i}}}^{T}\right)^{T}\left(\lambda^{i}-\overline{\lambda^{i}}\right)}
$$

where the bar represents the mean value of the vector. Note that, while we use 11 images to improve the estimation of the slope, two images are sufficient. The computation of the spectral decoder was carried out in Matlab 2019b.

Optimal linear estimator. Images acquired over time at the probing wavelength $\lambda_{\mathrm{p}}$ are first normalized to the average image over 10 acquisitions to establish a baseline. An image pixel $i$ in a sensor area with $N$ pixels has an associated (normalized) intensity value at time $\mathrm{k}$ given by $y_{k}^{i}$. The optimal linear estimator of the resonance shift at time $k$ over the sensor area is given by Eq. (2).

$$
\widehat{\Delta \lambda}_{k}=\frac{1}{\sum_{N}\left(\alpha^{i}\right)^{2}} \sum_{N} \alpha^{i}\left(1-y_{k}^{i}\right)
$$

For the utilized metasurfaces, the optimal linear estimator works well in the neighborhood of $\lambda_{p}$, which ranges from 2 to $3 \mathrm{~nm}$, depending on the spectral location of $\lambda_{p}$. For values outside the linear regime, we implemented a correction factor to enable the measurement of large spectral shifts (i.e., silica nanoparticles at high concentrations). All computations were carried out in Matlab 2019b.

Reporting summary. Further information on research design is available in the Nature Research Reporting Summary linked to this article.

\section{Data availability}

The data that support the plots within this paper and other findings of this study are available within the article, its supplementary information, or from the corresponding author upon reasonable request.

\section{Code availability}

The custom codes used in this study are available from the corresponding author upon reasonable request.

Received: 23 April 2020; Accepted: 12 April 2021; Published online: 31 May 2021

\section{References}

1. Borrebaeck, C. A. K. Precision diagnostics: moving towards protein biomarker signatures of clinical utility in cancer. Nat. Rev. Cancer 17, 199-204 (2017).

2. Paulovich, F. V., De Oliveira, M. C. F. \& Oliveira, O. N. A future with ubiquitous sensing and intelligent systems. ACS Sens. 3, 1433-1438 (2018).

3. Walper, S. A. et al. Detecting biothreat agents: from current diagnostics to developing sensor technologies. ACS Sens. 3, 1894-2024 (2018).

4. Yesilkoy, F. et al. Phase-sensitive plasmonic biosensor using a portable and large field-of-view interferometric microarray imager. Light Sci. Appl. 7, 17152-17152 (2018)

5. Belushkin, A. et al. Rapid and digital detection of inflammatory biomarkers enabled by a novel portable nanoplasmonic imager. Small 16, 1906108 (2020). 
6. Spackova, B., Wrobel, P., Bockova, M. \& Homola, J. Optical biosensors based on plasmonic nanostructures: a review. Proc. IEEE 104, 2380-2408 (2016).

7. Im, H. et al. Label-free detection and molecular profiling of exosomes with a nano-plasmonic sensor. Nat. Biotechnol. 32, 490-495 (2014).

8. Gomez-Cruz, J. et al. Cost-effective flow-through nanohole array-based biosensing platform for the label-free detection of uropathogenic $\mathrm{E}$. coli in real time. Biosens. Bioelectron. 106, 105-110 (2018).

9. Mazzotta, F. et al. Influence of the evanescent field decay length on the sensitivity of plasmonic nanodisks and nanoholes. ACS Photon. 2, 256-262 (2015).

10. Špačková, B., Ermini, M. L. \& Homola, J. High-performance biosensor exploiting a light guidance in sparse arrays of metal nanoparticles. Opt. Lett. 44, 1568-1571 (2019).

11. Vala, M., Ertsgaard, C. T., Wittenberg, N. J. \& Oh, S.-H. Plasmonic sensing on symmetric nanohole arrays supporting high-Q hybrid modes and reflection geometry. ACS Sens 4, 3265-3274 (2019).

12. Salemmilani, R., Moskovits, M. \& Meinhart, C. D. Microfluidic analysis of fentanyl-laced heroin samples by surface-enhanced Raman spectroscopy in a hydrophobic medium. Analyst 144, 3080-3087 (2019).

13. Soler, M. et al. Multiplexed nanoplasmonic biosensor for one-step simultaneous detection of Chlamydia trachomatis and Neisseria gonorrhoeae in urine. Biosens. Bioelectron. 94, 560-567 (2017)

14. Park, S.-H., Lee, J. \& Yeo, J.-S. On-chip plasmonic detection of microRNA106a in gastric cancer using hybridized gold nanoparticles. Sens. Actuat. B Chem. 262, 703-709 (2018).

15. Jackman, J. A., Rahim Ferhan, A. \& Cho, N.-J. Nanoplasmonic sensors for biointerfacial science. Chem. Soc. Rev. 46, 3615-3660 (2017).

16. Kaniber, M. et al. Surface plasmon resonance spectroscopy of single bowtie nanoantennas using a differential reflectivity method. Sci. Rep. 6, 23203 (2016).

17. Lee, H.-E. et al. Cysteine-encoded chirality evolution in plasmonic rhombic dodecahedral gold nanoparticles. Nat. Commun. 11, 263 (2020).

18. Oh, S.-H. \& Altug, H. Performance metrics and enabling technologies for nanoplasmonic biosensors. Nat. Commun. 9, 5263 (2018).

19. Tseng, M. L., Jahani, Y., Leitis, A. \& Altug, H. Dielectric metasurfaces enabling advanced optical biosensors. ACS Photon. https://doi.org/10.1021/ acsphotonics.0c01030 (2020).

20. Boriskina, S. V. et al. Losses in plasmonics: from mitigating energy dissipation to embracing loss-enabled functionalities. Adv. Opt. Photon. 9, 775 (2017).

21. Doiron, B. et al. Quantifying figures of merit for localized surface plasmon resonance applications: a materials survey. ACS Photon. 6, 240-259 (2019).

22. Kuznetsov, A. I., Miroshnichenko, A. E., Brongersma, M. L., Kivshar, Y. S. \& Luk'yanchuk, B. Optically resonant dielectric nanostructures. Science $\mathbf{3 5 4}$ aag2472 (2016).

23. Kruk, S. \& Kivshar, Y. Functional meta-optics and nanophotonics governed by mie resonances. ACS Photon. 4, 2638-2649 (2017).

24. Krasnok, A., Caldarola, M., Bonod, N. \& Alú, A. Spectroscopy and biosensing with optically resonant dielectric nanostructures. Adv. Opt. Mater. 6, 1701094 (2018).

25. van de Groep, J. \& Brongersma, M. L. Metasurface mirrors for external control of mie resonances. Nano Lett. 18, 3857-3864 (2018).

26. Koenderink, A. F., Alù, A. \& Polman, A. Nanophotonics: shrinking lightbased technology. Science 348, 516 (2015).

27. Rho, J. Metasurfaces: subwavelength nanostructure arrays for ultrathin flat optics and photonics. MRS Bull. 45, 180-187 (2020).

28. Genevet, P., Capasso, F., Aieta, F., Khorasaninejad, M. \& Devlin, R. Recent advances in planar optics: from plasmonic to dielectric metasurfaces. Optica 4 , 139-152 (2017).

29. Zhou, Y. et al. Multilayer noninteracting dielectric metasurfaces for multiwavelength metaoptics. Nano Lett. 18, 7529-7537 (2018).

30. Gholipour, B., Piccinotti, D., Karvounis, A., MacDonald, K. F. \& Zheludev, N. I. Reconfigurable ultraviolet and high-energy visible dielectric metamaterials. Nano Lett. 19, 1643-1648 (2019).

31. Bontempi, N. et al. Highly sensitive biosensors based on all-dielectric nanoresonators. Nanoscale 9, 4972-4980 (2017).

32. Yavas, O., Svedendahl, M., Dobosz, P., Sanz, V. \& Quidant, R. On-a-chip biosensing based on all-dielectric nanoresonators. Nano Lett. 17, 4421-4426 (2017).

33. Yavas, O., Svedendahl, M. \& Quidant, R. Unravelling the role of electric and magnetic dipoles in biosensing with Si nanoresonators. ACS Nano 13, $4582-4588$ (2019).

34. Hsu, C. W., Zhen, B., Stone, A. D., Joannopoulos, J. D. \& Soljačić, M. Bound states in the continuum. Nat. Rev. Mater. 1, 16048 (2016).

35. Koshelev, K., Bogdanov, A. \& Kivshar, Y. Meta-optics and bound states in the continuum. Sci. Bull. 64, 836-842 (2019)

36. Rybin, M. \& Kivshar, Y. Supercavity lasing. Nature 541, 164-165 (2017).

37. Koshelev, K., Lepeshov, S., Liu, M., Bogdanov, A. \& Kivshar, Y. Asymmetric metasurfaces with high- Q resonances governed by bound states in the continuum. Phys. Rev. Lett. 121, 193903 (2018).
38. Yesilkoy, F. et al. Ultrasensitive hyperspectral imaging and biodetection enabled by dielectric metasurfaces. Nat. Photon. 13, 390-396 (2019).

39. Yoshikawa, H. et al. Parallelized label-free detection of protein interactions using a hyper-spectral imaging system. Anal. Methods 7, 5157-5161 (2015).

40. Cetin, A. E. et al. Handheld high-throughput plasmonic biosensor using computational on-chip imaging. Light Sci. Appl. 3, e122-e122 (2014).

41. Lopez, G. A., Estevez, M.-C., Soler, M. \& Lechuga, L. M. Recent advances in nanoplasmonic biosensors: applications and lab-on-a-chip integration. Nanophotonics 6, 123-136 (2017)

42. Zhu, H., Mavandadi, S., Coskun, A. F., Yaglidere, O. \& Ozcan, A. Optofluidic fluorescent imaging cytometry on a cell phone. Anal. Chem. 83, 6641-6647 (2011).

43. Chiodi, E. et al. Highly multiplexed label-free imaging sensor for accurate quantification of small-molecule binding kinetics. ACS Omega 5, 25358-25364 (2020).

44. Kosaka, N. et al. Exploiting the message from cancer: the diagnostic value of extracellular vesicles for clinical applications. Exp. Mol. Med. 51, 1-9 (2019).

45. Keklikoglou, I. et al. Chemotherapy elicits pro-metastatic extracellular vesicles in breast cancer models. Nat. Cell Biol. 21, 190-202 (2019).

46. Sortino, L. et al. Enhanced light-matter interaction in an atomically thin semiconductor coupled with dielectric nanoantennas. Nat. Commun. 10, 5119 (2019).

47. Luo, X., Tsai, D., Gu, M. \& Hong, M. Extraordinary optical fields in nanostructures: from sub-diffraction-limited optics to sensing and energy conversion. Chem. Soc. Rev. 48, 2458-2494 (2019).

48. Ziolkowski, R. W. \& Engheta, N. Metamaterials: two decades past and into their electromagnetics future and beyond. IEEE Trans. Antennas Propag. 68, $1232-1237$ (2020)

49. Rippa, M. et al. Octupolar plasmonic nanosensor based on ordered arrays of triangular Au nanopillars for selective rotavirus detection. ACS Appl. Nano Mater. https://doi.org/10.1021/acsanm.0c00872 (2020).

50. Jackman, J. A. et al. Plasmonic nanohole sensor for capturing single virus-like particles toward virucidal drug evaluation. Small 12, 1159-1166 (2016).

51. IUPAC. Compendium of Chemical Terminology: Gold Book (IUPAC, 2009).

52. Mathieu, M., Martin-Jaular, L., Lavieu, G. \& Théry, C. Specificities of secretion and uptake of exosomes and other extracellular vesicles for cell-to-cell communication. Nat. Cell Biol. 21, 9-17 (2019).

53. Zhang, P. et al. Ultrasensitive detection of circulating exosomes with a 3Dnanopatterned microfluidic chip. Nat. Biomed. Eng. 3, 438-451 (2019).

54. Andreu, Z. \& Yáñez-Mó, M. Tetraspanins in extracellular vesicle formation and function. Front. Immunol. 5, 442 (2014).

55. Galindo-Hernandez, O. et al. Elevated concentration of microvesicles isolated from peripheral blood in breast cancer patients. Arch. Med. Res. 44, 208-214 (2013).

56. Moloney, B. M. et al. Investigating the potential and pitfalls of EVencapsulated microRNAs as circulating biomarkers of breast cancer. Cells $\mathbf{9}$, 141 (2020).

57. Johnsen, K. B., Gudbergsson, J. M., Andresen, T. L. \& Simonsen, J. B. What is the blood concentration of extracellular vesicles? Implications for the use of extracellular vesicles as blood-borne biomarkers of cancer. Biochim. Biophys. Acta Rev. Cancer 1871, 109-116 (2019).

\section{Acknowledgements}

The authors thank T. Andrejic and S. Taghishokrgozar for assistance in preparing the sensor chips, École Polytechnique Fédérale de Lausanne, and Center of MicroNano Technology (CMi) for nanofabrication. We also acknowledge funding from the European Union Horizon 2020 Framework Programme for Research and Innovation under Grant Agreements No.FETOPEN-737071 (ULTRA-CHIRAL Project) and no. 777714 (NOCTURNO project), the European Research Council under grants agreement no. 682167 (VIBRANT-BIO) and no. 725051 (EVOLVE), the Australian Research Council (the grant DP210101292), the Strategic Fund of the Australian National University, and the Russian Foundation for Basic Research (19-02-00419). K. Koshelev thanks the Advancement of Theoretical Physics and Mathematics "BASIS".

\section{Author contributions}

Y.J., E.R.A., and H.A. conceived and designed the research. Y.J. designed and fabricated the dielectric metasurfaces. Y.J., F.Y., and E.R.A. built the measurement setup. Y.J. carried out the experiments. E.R.A. composed the code and analyzed data. Y.K. and K.K. contributed to the theoretical analysis of the metasurfaces. C.C. and M.D.P. provided the biosamples. All authors contributed to the writing of the manuscript.

\section{Competing interests}

The authors declare no competing interests. 


\section{Additional information}

Supplementary information The online version contains supplementary material available at https://doi.org/10.1038/s41467-021-23257-y.

Correspondence and requests for materials should be addressed to H.A.

Peer review information Nature Communications thanks Jong-Souk Yeo and Stefano Cabrini for their contribution to the peer review of this work.

Reprints and permission information is available at http://www.nature.com/reprints

Publisher's note Springer Nature remains neutral with regard to jurisdictional claims in published maps and institutional affiliations. (c) (1) Open Access This article is licensed under a Creative Commons Attribution 4.0 International License, which permits use, sharing, adaptation, distribution and reproduction in any medium or format, as long as you give appropriate credit to the original author(s) and the source, provide a link to the Creative Commons license, and indicate if changes were made. The images or other third party material in this article are included in the article's Creative Commons license, unless indicated otherwise in a credit line to the material. If material is not included in the article's Creative Commons license and your intended use is not permitted by statutory regulation or exceeds the permitted use, you will need to obtain permission directly from the copyright holder. To view a copy of this license, visit http://creativecommons.org/ licenses/by/4.0/.

(C) The Author(s) 2021 